\title{
Intelligent Model for Manual Sorting of Plastic Wastes
}

\author{
Kudirat Jimoh \\ Computer Sc. \& Eng. Dept., \\ Obafemi Awolowo University, \\ lle-Ife, Nigeria
}

\author{
Anuoluwapo Ajayi \\ Computer Sc. \& Eng. Dept., \\ Obafemi Awolowo University, \\ lle-Ife, Nigeria
}

\author{
Oluwatobi Ayilara \\ Computer Sc. \& Eng. Dept., \\ Obafemi Awolowo University, \\ lle-Ife, Nigeria
}

\begin{abstract}
The need for an automated sorting system cannot be overemphasized due to the growing needs for high throughput and accuracy. Manual sorting method may achieve good accuracy, but at the expense of throughput. This study formulates an automated plastic wastes identification model to overcome the limitation of the manual sorting method. The proposed identification model employed singleton Sugeno fuzzy model, image processing and template matching techniques as its classifier using physical properties of plastics such as power spectrum of sound, plastic average area and plastic recycling code as feature set. The model was developed and simulated in the MATLAB R2012a environment. The performance evaluation of the model was carried out using three performance metrics, namely, accuracy, precision and recall. The model obtained average accuracy of 0.88 , average precision value of 0.77 and average recall value of 0.25 , respectively for the plastic types (PET, HDPE, LDPE, and PP) used in this study.
\end{abstract}

\section{General Terms}

Plastic identification, Fuzzy logic, Template matching, spectral analysis, Image processing.

\section{Keywords}

Plastic wastes, inference system, sorting, resin code.

\section{INTRODUCTION}

The amount of plastic waste (sachet water bags, plastic bottles, wrapping products, and so on) is on the increase in many countries due to the increase in population. In developing countries, for instance, the absence of a sound policy to ensure timely collection, transportation and disposal of plastic wastes has encouraged people to dispose plastic wastes on streets with impunity. These wastes find their way in blocked channels, thereby causing floods and other environmental hazards. The need for a model to identify plastic wastes for efficient recycling process is thus desirable. Sorting of plastic resins is an important step in plastic recycling and many wide ranges of incompatible plastic resins abound that may inhibit recycling process. The separation of different plastics by type is mandatory because contamination in the recycling of one type of plastic with another can cause processing problems [10].

Methods for sorting waste plastics range from manual sorting to advanced automated technologies. The sorting and separation techniques are often done using chemical, optical, electrical or physical property differences between various plastics to be sorted [15]. Density based sorting methods include float-sink tanks and hydrocyclone. Optical and spectroscopic-based methods were used to separate plastics on the basis of colour [13], [19] or type [12], [16-17]. These techniques cannot identify different grades of the same polymers and cannot be used when plastic waste surface is wet. [1] developed an automatic sorting of plastics based on optical identification of fluorescence signatures of dyes, incorporated into such materials in trace concentrations. This work's limitation is the speed and purity of sorting, which were limited by the mechanical singulation inadequacy in the conveyor system at high speeds.

Auto-sort technologies based on X-ray detection [9] was used to differentiate between PVC and PET bottles as X-ray sensors was able to detect the presence of chlorine atom found in PVC bottles. This method cannot identify other plastic resin types, besides, X-rays are a form of radiation; hence, precautions must be taken to protect workers from exposure. In [15], a probabilistic approach was proposed to sort plastic wastes into PET and non-PET by integrating size, color and distance. White strips on bottle are first identified by employing maximum likelihood approach and information on the white and grey strips is then analyzed by using maximum a posteriori method.

Other research conducted on the use of artificial neural networks in conjunction with infrared spectroscopy for efficient sorting of plastics include [4], [7]. [3] proposed an Xray fluorescence detection system based on the use of tracers for plastics identification while research based on machine vision or image processing technique for plastic bottles identification are discussed in [14], [24].

Truly, automated sorting systems can treat large volume of plastic efficiently with minimal human intervention but they often require high investments in specific technologies [6], [21]. Manual sorting on the other hand, relies on plant personnel for visual identification and manual extraction of the plastic containers. However, this is not suitable for plants with large throughput as efficiency of the process is reduced. Nevertheless, in many developing countries human labour is cost effective when compared to investment required for automated systems, more so manual sorting in recycling plants is still the preferred procedure [23]. It is therefore necessary to improve the manual process of sorting polymer materials so as to make their recycling profitable. For an economically efficient recycling of plastic wastes, waste plastics need to be sorted cheaply and automatically into individual types and grades [5]. This study therefore, proposes a model, which combined fuzzy logic model, image processing technique with template matching algorithm to automate the manual sorting method so as to overcome its limitations (accuracy and throughput) as well as improve its efficiency.

The category of plastic materials used in this study are limited to polyethylene terephthalate (PET), high density polyethylene (HDPE), low density polyethylene (LDPE) and polypropylene (PP).

The rest of this paper is organized as follows. Section 2 describes the proposed plastic wastes identification model. 
Simulation environment and performance evaluation of the model are discussed in sections 3 and 4. Finally, concluding remarks are given in section 5 .

\section{IDENTIFICATION MODEL}

Classification problem involves associating an object with existing classes of objects. Classes may be defined as a set of objects, or by a set of rules, which define how objects are classified into given classes. In this study, the classification of plastic wastes considered only the physical properties so as to reduce implementation cost. The distinguishing physical characteristics used for classification purpose are power spectrum of sound, plastic recycling symbol code and plastic average area.

Two classification schemes were employed in this study. The first scheme used template matching to identify plastic types having the recycling symbol (labeling system instituted by the Society of the Plastics Industry) inside a triangle printed at the bottom of the bottle. However, most plastics manufactured locally are devoid of this symbol and even when present, may be indistinct. The singleton fuzzy method, which is based on using fuzzy rules, is employed as a classification scheme for cases of this nature. Figure 1 depicts a simplified logic of the proposed plastic wastes identification model. Captured plastic image and sound clip are input to this model. The model first tries to locate the recycling symbol on plastic. If present, the template matching algorithm is used to determine appropriate plastic type. Otherwise, distinguishing physical characteristics (spectrum power of the plastic sound and plastic area) are then computed and fed into the Singleton fuzzy model to determine plastic type. Key modules of this model are discussed in the subsections following.

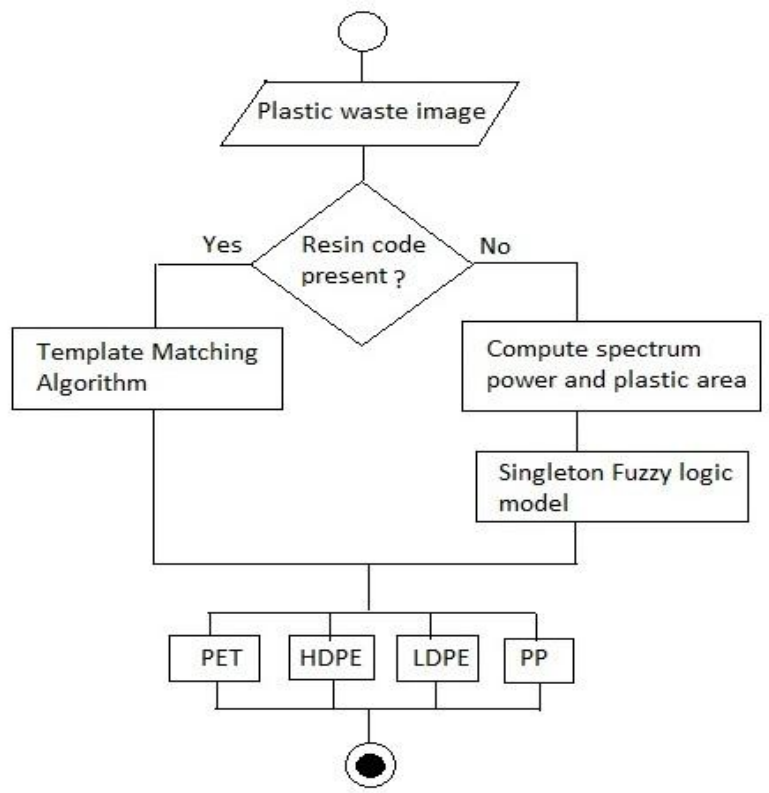

Fig 1:Plastic wastes identification model

\subsection{Image Processing}

The preprocessing operation reduces noise and redundant data for the purpose of acquiring an optimum-quality image that will enhance the classifier performance. The preprocessing operations (image size reduction, image enhancement, morphology were carried out on the acquired images to extract necessary features. The main purpose of feature extraction is to represent the data in a reduced number of dimensions so as to removing redundancy and improve classification. Features such as area, filling fraction of the boundary box measured in pixel units, and the sound produced by tapping or rubbing the plastic surface were recorded. Sound was used as a distinguishing feature since most plastics produce unique sound to uniquely identify them. For instance; nylon, a specific type of LDPE resin, produces a unique sound when compared to other resins when squeezed.

\subsection{Template Matching}

Template matching is a technique used in classifying an object by comparing portions of images with another image. It is the simplest and widely used for detecting targets in image information [2]. The template matching problem is described as follows: Assign an unknown target $\mathrm{Y}$ into one of $\mathrm{C}$ classes, which are represented by the specified templates $\left\{X_{1}\right\}$ for $1=1,2 \ldots, C$. The matching process consists of defining a similarity measure between the objects $\mathrm{Y}$ and $\mathrm{X}_{\mathrm{l}}$, denoted by $\mathrm{S}\left(\mathrm{Y}, \mathrm{X}_{\mathrm{l}}\right)$ and assigning object $\mathrm{Y}$ to class $\mathrm{k}$ if $\mathrm{S}\left(\mathrm{Y}, \mathrm{X}_{\mathrm{k}}\right)>\mathrm{S}(\mathrm{Y}$, $\mathrm{X}_{\mathrm{l}}$ ) for all $1 \neq \mathrm{k}$. This procedure was used to classify resin codes in plastic wastes using the feature-based approach, an approach well suited for cases where both reference and template images have more correspondence with respect to features and control points.

Plastic resin code samples are first predefined and stored in the database. Then, the correlation between the input image blocks and stored resin code samples are computed to identify plastic type. The advantages of this method is that it is robust to noise, simple to implement and it takes less time to locate the candidate resin from input images. Template matching was accomplished with a $24 \times 24$ pixel templates representing plastic resin codes of plastic waste images (where present). Matching technique also calculates the error between input image and stored resin codes templates using the mean squared error (MSE) metric:

$$
M S E=\frac{\sum \sum \text { template }(x, y)-\text { reference }(x, y)}{\text { Number of pixels }}
$$

where, template $(\mathrm{x}, \mathrm{y})$ and reference $(\mathrm{x}, \mathrm{y})$ are the intensity values of template and input images, respectively. The templates of all the resin codes will be define with some test point. Given an image, the resin code to be recognized will be compare with those templates, and the template with the highest matched point will be the resin code that appears in that image.

\subsection{Fuzzy Logic Model}

The fuzzy inference method used in this system is the TakagiSugeno-Kang (TSK) [18], [20]. Fuzzy rules represent classes in 'fuzzy' terms using linguistic variables. This technique enables approximate reasoning to be performed. The technique also improve performance of classification systems in three ways: through efficient numerical representation of vague terms and concepts, by increasing their range of operation in ill-defined environments, and by decreasing their sensitivity to noisy data. Fuzzy logic-based systems operate based on the precise and rigorous mathematics of fuzzy sets. A fundamental concept of fuzzy sets is that an element $\mathbf{x}$ may be a member of set $\mathbf{A}$ with varying degrees, i.e., each member of the set is characterized by its degree of membership within the set. The degree of membership of element $\mathbf{x}$ in set $\mathbf{A}$ is denoted by $\mu_{A}(x)$. A mapping of the domain interval to its degree of membership defines the membership function $\mu$. The basic structure of a fuzzy inference system consists of the following: (1) the fuzzifier, which converts the crisp inputs into fuzzy inputs, (2) a knowledge base, which contains fuzzy 
rules along with membership functions (3) an inference mechanism that applies a fuzzy reasoning mechanism to derive a fuzzy output; and (4) a defuzzifier, which translates the fuzzy output into a crisp value. The Singleton model, which is a special case of Mamdani or TSK fuzzy models, was used due to less computational efforts of its defuzzification process when compared to Mamdani's fuzzy inference method [11]. Besides, the Mamdani model lack accuracy and has high computational cost [22]. The fuzzy rule for the Singleton model is of the form:

$\mathrm{R}_{\mathrm{i}}$ : If $\mathrm{x}_{1}$ is $\mathrm{A}_{\mathrm{i} 1}$ and ....... and $\mathrm{x}_{\mathrm{n}}$ is $\mathrm{A}_{\mathrm{in}}$ then $\mathrm{y}$ is $\mathrm{C}$

where, $\mathrm{C}$ is a fuzzy singleton.

Each linguistic variable is usually associated with a complete set of membership function that is defined over the entire operating range of that variable. Figure 2 shows examples of membership functions set for the two input variables used in this study - average power spectrum of sound produced from plastic ("averageSpectrumPower") and the average plastic area ("averageShapeArea"). The membership functions are triangular functions with the widths of membership functions appropriately determined by the dispersion of the data within the membership range. The triangular curve is a function of a vector $\mathrm{x}$, and depends on scalar parameters: $\mathrm{a}, \mathrm{b}$ and $\mathrm{c}$, as given by.

$f(x: a, b, c)=\left\{\begin{array}{cc}0, & x \leq a \\ (x-a) /(b-a), & a \leq x \leq b \\ (c-x) /(c-b), & b \leq x \leq c \\ 0, & c \leq x\end{array}\right\}$

The range of the membership functions for input "averageSpectrumPower" is from 0 to $3.4 \mathrm{e} 10-5$, while the range for input variable "averageShapeArea" is normalized with the biggest plastic area size value set as 1 (x-axis) and the smallest plastic area size set as 0 (x-axis). Subsection 3.4 described how the spectrum power of sound generated from plastic wastes were computed. Six membership functions selected for plastic's average spectrum power were 'Zero $(\mathrm{Z})$ ', 'Very Low (VL)', 'Low (L)', 'Medium (M)', 'High (H)' and 'Very High (VH)'. The five membership functions selected for plastic's average area were 'Very Small (VS)', 'Small (S)', 'Medium (M)', 'Big (B)' and 'Very Big (VB)'.

The number of membership functions and the shape of these functions are an essential part of the knowledge embodied in a fuzzy logic system. The domain expert usually supplies this information. This information when combined with the rules forms a complete knowledge-base for a given application. After a thorough observation of the data set, the rules were constructed as showed in Table 1. The minimum aggregation method is used in the aggregation of the rules in this study to form the aggregate output of the fuzzy set.

The defuzzification method used in the fuzzy system is weighted average. The output of the fuzzy singleton model is the plastic type (PET, HDPE, LDPE, and PP). The fuzzy system used to identify plastic wastes without resin codes accepts average power spectrum and average plastic area as inputs. The output is a single crisp number, which represents the aggregation and deffuzzication of the inputs. The fuzzy systems used to process plastic images with their corresponding sound clips is represented in Figure 4.

Figure 5 illustrates the rules viewer given example inputs: aveSpectrumPower $=1.75 \mathrm{e}-05 \mathrm{~dB} / \mathrm{Hz}$ and aveShapeArea $=$ 0.423 meters, with the corresponding output as plasticType $=$
2, signifying the resin type of HPDE. The rules' inferencing system is shown in the viewer as well.
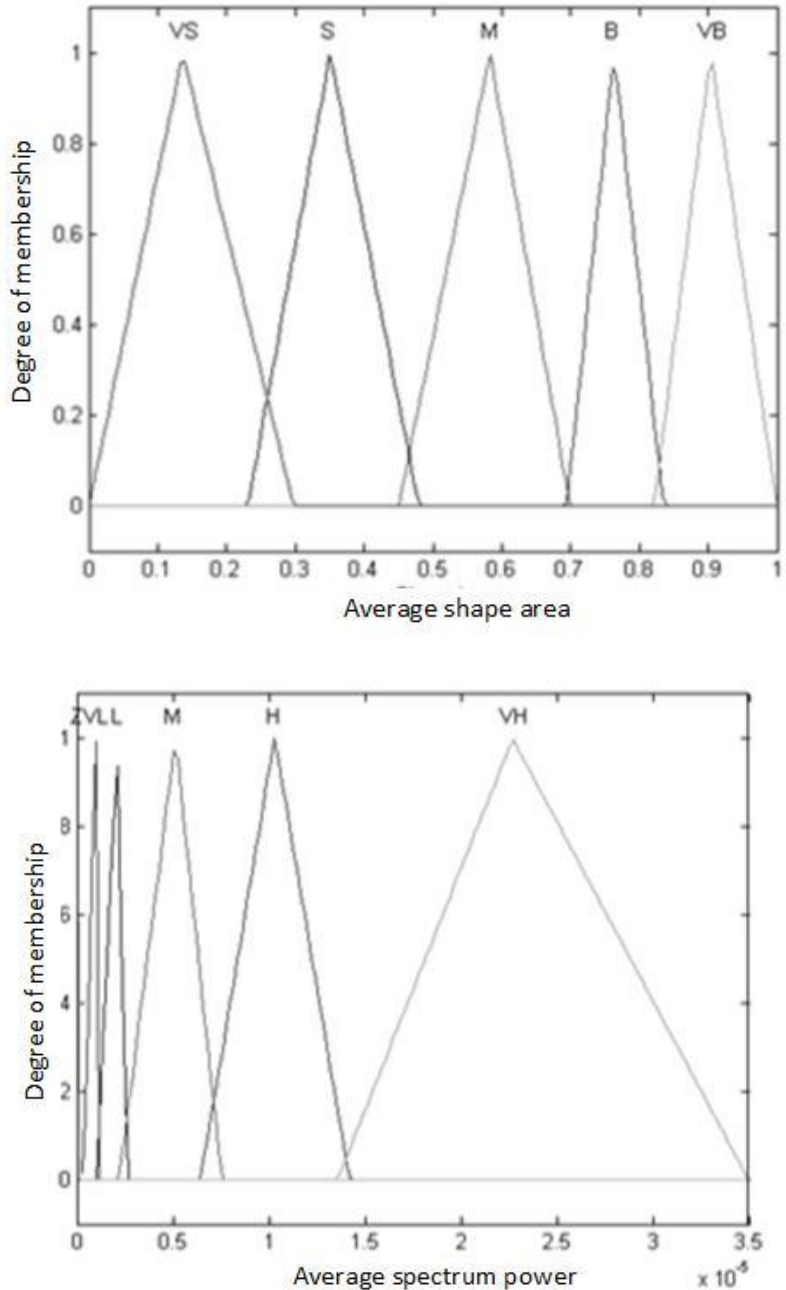

Fig 3: Input variables membership functions

Table 1. Fuzzy logic rule set

\begin{tabular}{|c|c|c|c|c|c|}
\hline $\begin{array}{l}\text { Ave. Area/ } \\
\text { Ave. Spectrum } \\
\text { power }\end{array}$ & None & S & M & B & VB \\
\hline Z & - & PP & PP & PP & HDPE \\
\hline VL & LDPE & LDPE & LDPE & LDPE & LDPE \\
\hline L & - & PP & PET & PET & PET \\
\hline M & - & - & PP & PET & - \\
\hline H & - & PP & PET & PET & HDPE \\
\hline VH & - & HDPE & PET & PET & HDPE \\
\hline
\end{tabular}




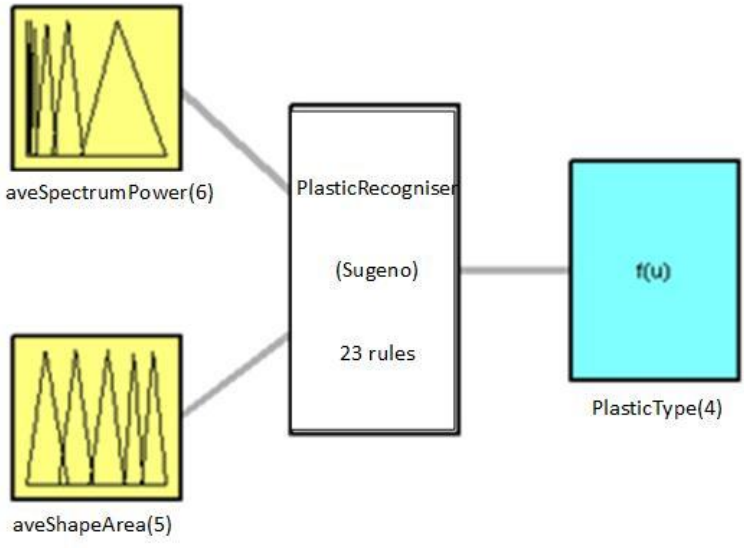

Fig 4: Fuzzy inference system

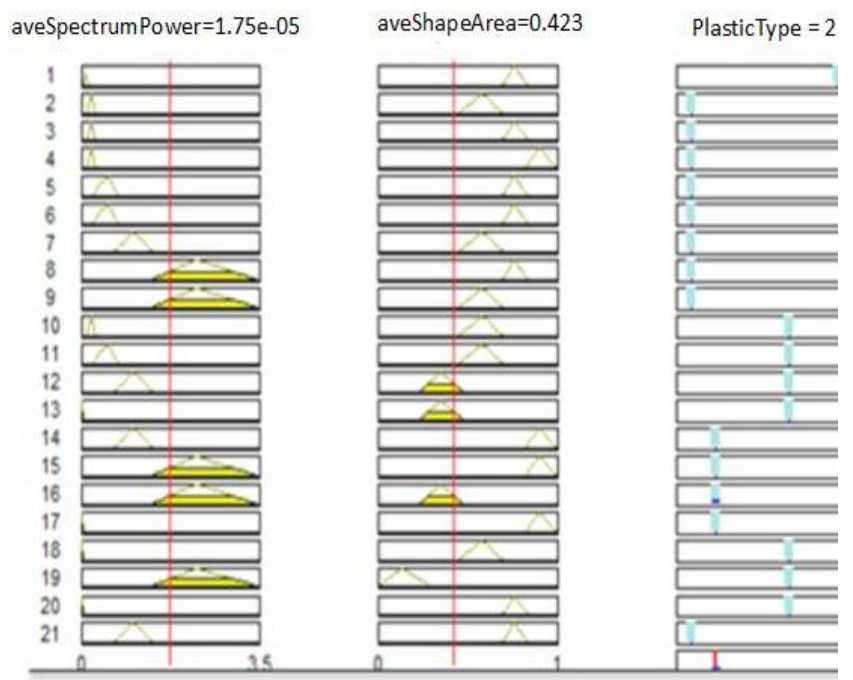

Fig 5: Rules viewer with input examples

\subsection{Spectrum Power Computation}

The spectral analysis of sound produced by a given plastic waste sample is determined as follows. The average power of a deterministic periodic signal is computed using the following: The power spectral density (PSD) of a stationary random process $x_{n}$, in terms of normalized frequency, is given as

$$
P_{x x}(\omega)=\frac{1}{2 \pi} \sum_{m=-\infty}^{\infty} R_{x x}(m) e^{-j \omega m}
$$

Eq (1) when written as a function of a physical frequency $f$ (using the relation $\omega=2 \pi f / f_{s}$, where $f_{s}$ is the sampling frequency) gives

$$
P_{x x}(f)=\frac{1}{f_{s}} \sum_{m=-\infty}^{\infty} R_{x x}(m) e^{-j \pi m f / f_{x}}
$$

The correlation sequence from the PSD is then derived using the inverse discrete-time Fourier transform given as:

$$
\begin{aligned}
R_{x x}(m)=\int_{-\pi}^{\pi} P_{x x}(\omega) e^{j \omega m} d \omega & \\
= & \int_{-f_{x} / 2}^{f_{x} / 2} P_{x x}(f) e^{j 2 \pi m f / f_{x}} d f
\end{aligned}
$$

Finally, the average power of the sequence $x_{n}$ over the entire Nyquist interval is computed using

$$
R_{x x}(0)=\int_{-\pi}^{\pi} P_{x x}(\omega) d \omega=\int_{-f_{x} / 2}^{f_{x} / 2} P_{x x}(f) d f
$$

The above expression $\operatorname{Pxx}(\omega)$ represents the power content of a signal in an infinitesimal frequency band.

The average power of a signal over a particular frequency band [ $\omega 1, \omega 2], 0 \leq \omega 1 \leq \omega 2 \leq \pi$, is found by integrating the PSD over that band.

Periodogram is the simplest nonparametric method used to estimate PSD directly from the signal itself. In general terms, one way of estimating the PSD of a process is to simply find the discrete-time Fourier transform of the samples of the process (usually done on a grid with an FFT) and appropriately scale the magnitude squared of the result. This estimate is called the periodogram. The computation of the periodogram via an FFT algorithm in some cases, is more efficient if the number of frequencies is a power of two. Therefore it is not uncommon to pad the input signal with zeros to extend its length to a power of two. The periodogram estimate of the PSD of a sample LDPE sound signal used in this study, was computed by multiplying its data vector by a Hamming window. This is as depicted in Figure 6.

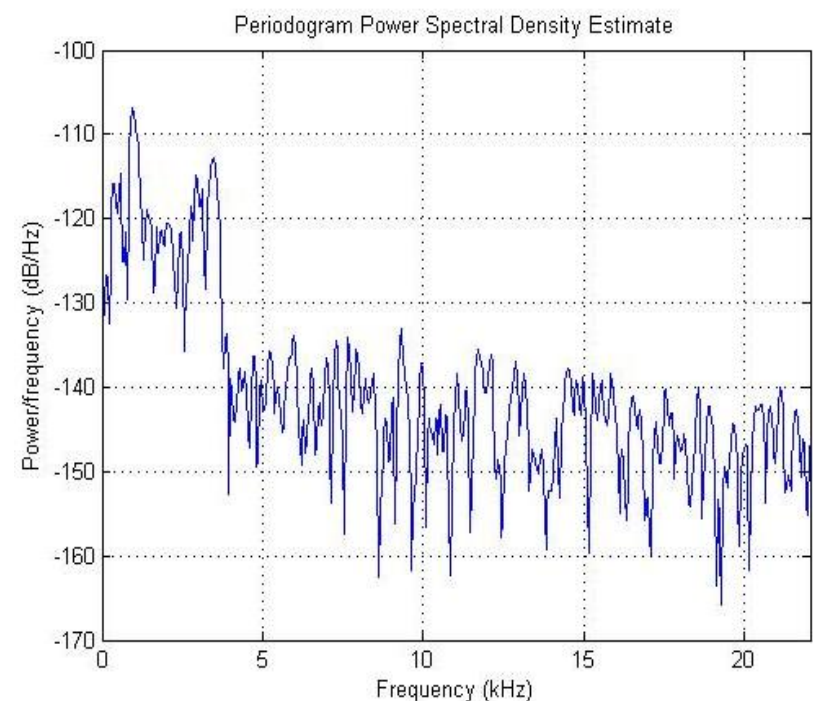

Fig 6: Rules viewer with input examples

\section{MODEL SIMULATION}

The plastic wastes identification model was simulated in MATLAB R2012a environment. Sample plastic wastes data (rescaled to size 90 by 110) with their equivalent wave sounds (file size between $3 \mathrm{~KB}$ to $5 \mathrm{~KB}$ ) were processed on an Intel Core i5 $2.50 \mathrm{GHz}$ machine using the model proposed in this study. MATLAB software was adopted because of its user friendliness, scalability and many other support features it provides.

\subsection{Data Sample Used}

For the plastic sorting model, image samples of PET, HDPE, LDPE and PP were collected.

In total, 500 samples from streets and disposal sites were collected for the test. Sample data including bottles and containers with different sizes consisted of PET (35\%), $\operatorname{HDPE}(20 \%)$, LDPE(30\%) and PP(15\%), respectively.

Brief description of the resins used in this study follows: 
i). Polyethylene Terephthalate (PET No. 1) containers. They are commonly used for beverages such as mineral water and soda beverages. These items are identified as always being transparent and usually green or clear in color.

ii). High Density Polyethylene (HDPE No. 2) containers. They are either rigid or soft. Rigid HDPE containers are opaque and solid in color, such as oil containers, household cleaning solution bottles, base cups of large beverage bottles, etc. Soft HDPE containers (such as juice and milk bottles) are semi-transparent and white colored.

iii). Low Density Polyethylene (LDPE No. 4). These are simply film plastics used for such purposes as agricultural covering, grocery bags, food industry wrap, dry cleaning bags, and so on.

iv). Polypropylene (PP No. 5). These are plastics used in the following products: battery cases, medical containers, oil additive containers, some dairy tubs, cereal box liners, bottle labels and caps, rope and strapping, combs, snack wraps, and bags.

\section{PERFORMANCE EVAlUATION}

The classification results is shown in graphical form (bar and pie charts) for easy interpretation. Figure 3 depicts sample output indicating the model's accuracy in plastic identification problem. Fig. 3(a) is a sample input of plastic images consisting of plastics with recycling code and those without recycling code. Outputs from the model are presented in two forms- bar chart and pie chart. The bar chart (Fig. 3b) shows the classification of each plastic sample into the appropriate resin type (PET, HDPE, LDPE, and PP), while the pie chart (Fig. 3c) depicts the frequency of each plastic type. The three metrics used to evaluate the performance of this model are accuracy $(A)$, precision $(P)$ and recall $(R)$.

Given that $T n$ is the total number of sampled plastics, $t p$ is the true positive detection of a specific plastic type, $f p$ is the false positive detection of a particular plastic type, tn is the true negative detection of a given plastic type and $f n$ is the false negative detection of a given plastic type, then

$$
\begin{gathered}
A=\frac{t p+t n}{T_{n}} \\
P=\frac{t p}{t p+f p} \\
R=\frac{t p}{t p+f n}
\end{gathered}
$$

To evaluate the effectiveness of our model to recognize plastic types used in this study, we made experiments in which random sample of plastic images (those with resin codes and those without resin codes) with their respective sound signals where applicable, are presented to the model as input. The classification efficiency of the model using performance metrics defined above was recorded. Table 2 shows the performance of the model to recognize plastic types used in this study. The accuracy of the model are 0.88 (PET), 0.86 (HDPE), 0.93 (LDPE) and 0.89(PP), respectively. The average accuracy $(0.89)$, precision $(0.77)$ and recall $(0.25)$ values obtained in this model can be attributed to the use of simple but efficient algorithms, in addition to the feature data utilized for identification purpose.

Table 2. Performance evaluation of the model

\begin{tabular}{|c|c|c|c|}
\hline Resin Type & A & P & R \\
\hline PET & 0.92 & 0.73 & 0.25 \\
\hline HDPE & 0.86 & 0.75 & 0.28 \\
\hline LDPE & 0.93 & 0.93 & 0.27 \\
\hline PP & 0.82 & 0.66 & 0.20 \\
\hline Average & 0.88 & 0.77 & 0.25 \\
\hline
\end{tabular}

\section{CONCLUSION}

In this study, a singular fuzzy logic model, template matching and image processing techniques were applied to automate the manual process of sorting and identifying four plastic types (PET, HDPE, LDPE, and PP). The use of fuzzy logic as an alternative to other intelligent techniques such as neural networks and genetic algorithms provides a computational inexpensive alternative.

The system developed in this study attempts to emulate the manual process of plastic wastes identification using physical properties such as resin code, area and sound wave in a more timely and effective manner. The model was developed and its performance evaluated. It was observed that the model was capable of classifying plastic wastes using physical characteristics such as sound clips, recycling code, and plastic area of plastics. It is also observed that sound signal can be used in plastic waste identification.

This model can be used to separate plastic wastes before they are sent to landfill sites to lower cost implication. Since most of the current commercial technologies have process capabilities and price ranges that limit their use to large volume recyclers, in future, we intend to build a robot that will incorporate the model developed in this study as its vision, and used in place of human to optimize plastic wastes recycling process. 


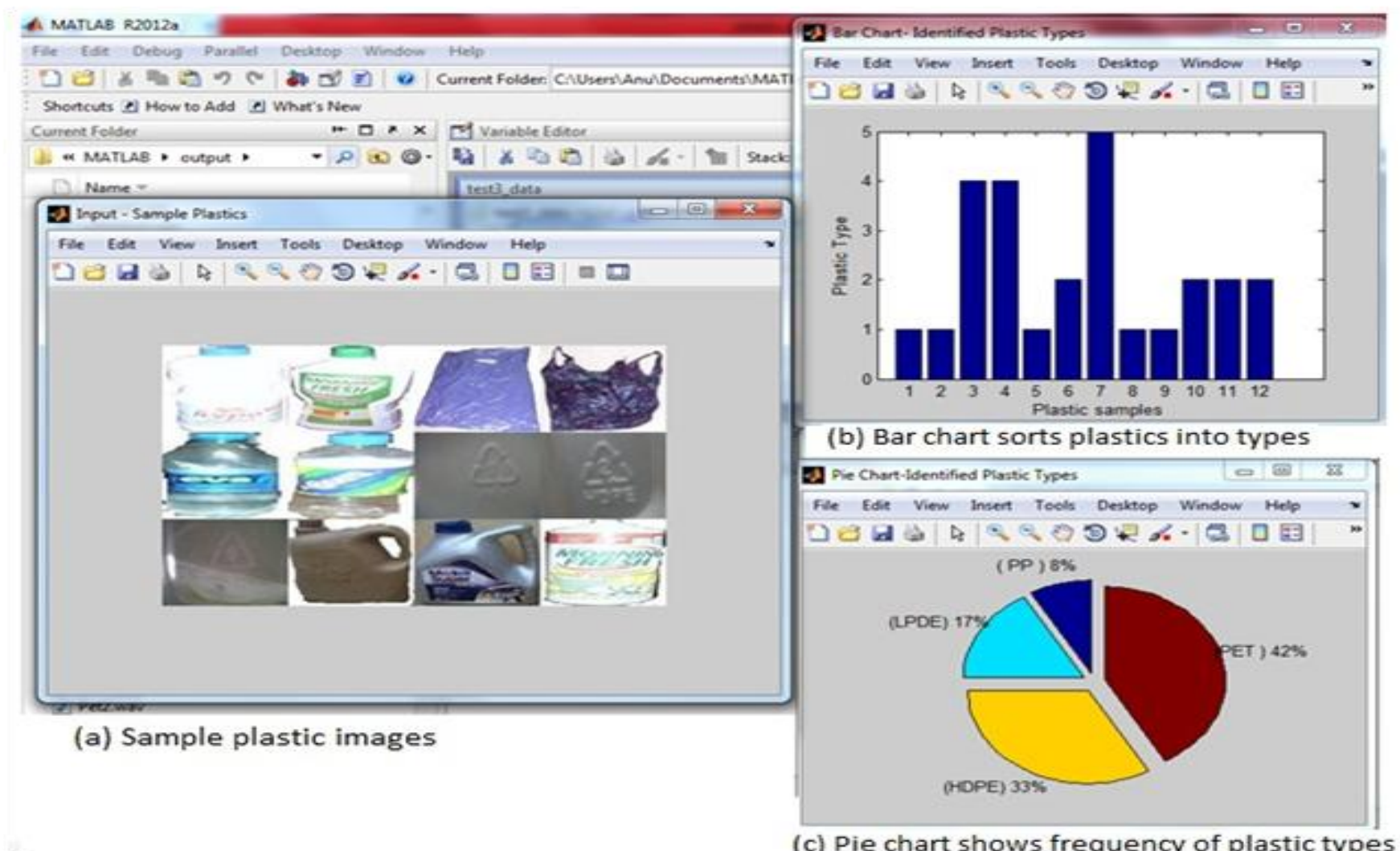

Fig 5: Classification results (b and c) for the sample plastic images in (a) as input

\section{REFERENCES}

[1] Ahmad, S.R., 2004. A new technology for automatic identification and sorting of plastics for recycling. Environ. Technol. 25 (10), 1143-1149.

[2] Bae, J.S and Song, T.L. 2008. Image Tracking Algorithm using Template Matching and PSNF-m. International Journal of Control, Automation, and Systems, 6(3), 413423

[3] Bezati, F. Froelich, D. Massardier, V. Maris, E. 2010. Addition of tracers into the polypropylene in view of automatic sorting of plastic wastes using X-ray fluorescence spectrometry, Waste management, 30,591596

[4] Coghlan, A. 1993. Infrared Makes Light Work of Sorting Plastics. New Scientist, 139(1882), 20.

[5] Froelich, D., Maris, E., Haoues, N., Chemineau, L., Renard, H., Abraham, F., Lassartesses, R. 2007. State of the art of plastic sorting and recycling: feedback to vehicle design. Miner. Eng. 20 (9 SPEC. ISS.), pp. 902912

[6] Hearn, G.L., Ballard, J.R. 2005. The use of electrostatic techniques for the identification and sorting of waste packaging materials. Resour. Conserv. Recycl., 44(1):9198

[7] Huth-Fehre, T. Feldhoff, R. Kantimm, T. Quick, L. Winter, F., Cammann, K., van den Broek, W. Wienke, D., Melssen, W. and Buydens, L. 1995. NIR-Remote Sensing and Artificial Neural Networks for Rapid Identification of Post Consumer Plastics. Journal of Molecular Structure, 348, 143-146.
[8] Hayes, M.H. Statistical Digital Signal Processing and Modeling. New York: John Wiley \& Sons, 1996.

[9] Inculet, I. 1992. Electrostatic separation of mixed plastic waste Proc. Davos 92 Recycle

[10] Masoumi H, Safavi S. M., and Khani Z. 2012. Identification and Classification of PlasticResins using Near Infrared ReflectanceSpectroscopy. International Journal of Mechanical and Industrial Engineering 6,213220

[11] Mclvor, R.T, McCloskey, A.G, Humphreys, P.K, Maguire, L.P. 2004. Using a fuzzy approach to support financial analysis in the corporate acquisition process. Expert Systems with Applications, 27,533-547

[12] Moore, S. 1999. Infrared scanner affords easy plastic identification. Mod. Plast. 76, 32-33.

[13] Safavi, S.M., Masoumi, H., Mirian, S. S., Tabrizchi, M. 2010. Sorting of polypropylene resins by color in MSW using visible reflectance spectroscopy. Waste Management 30, 2216-2222.

[14] Scavino, E., Wahab, D.A. Hussain, A. Basri, H. and Mustafa. M.M. 2009. Application of automated image analysis to the identification and extraction of recyclable plastic bottles. Journal of Zhejiang University of Science A, 10(6):794-799

[15] Scheirs, J. 1998. Polymer Recycling, Wiley Publication,

[16] Scott, D. M. 1995. A Two-Colour Near-Infrared Sensor for Sorting Recycled Plastic Waste. Measurement Science and Technology, 6(2), $156-159$.

[17] Sommer, E. J. 1996. Methods and technologies for postconsumer plastic identification and sorting. Proc. SPE 3rd Annual Recycling Conf., Chicago, IL, pp. 299-304. 
[18] Sugeno, M. and Kang, G.T. 1988. Structure identification of fuzzy model, Fuzzy Sets and Systems, $28,15-33$.

[19] Tachwali, Y. Al-Assaf, Y. Al-Ali, A.R. 2007. Automatic multistage classification system for plastic bottles recycling, Resources, Conservation \& Recycling. 52(2), 266-285

[20] Takagi, T. and Sugeno, M. 1985. Fuzzy identification of systems and its applications to modeling and control, IEEE transactions on Systems, Man, and Cybernetics 15, 116-132.

[21] Taylor, B. 2006. Visions of plastic. Recycl. Today, 44(4), 48-56

[22] Wadhawan, S., Goel G. and Kaushik S. 2013. Data Driven Fuzzy Modeling for Sugeno and Mamdani Type
Fuzzy Model using Memetic Algorithm .Information Technology and Computer Science, 8, 24-37

[23] Wahab, D.A., Abidin, A., Azhari, C.H. 2007. Recycling trends in the plastics manufacturing and recycling companies in Malaysia. J. Appl. Sci., 7(7), 1030-1035.

[24] Wahab, D.A., Hussain, A., Scavino, E., Mustafa, M.M., Basri, H. 2006. Development of a prototype automated sorting system for plastic recycling. Am. J. Appl. Sci., 3(7), 1924-1928.

[25] Zulkifley, M. A, Mustafa, M. M and Hussain, A. 2013. Probabilistic White Strip Approach to Plastic Bottle Sorting System, IEEE International Conference on Image Processing, Melbourne, Australia. pp: 3162-3166. 